\title{
Severe cerebral edema following nivolumab treatment for pediatric glioblastoma: case report
}

\author{
Xiao Zhu, BA, ${ }^{1}$ Michael M. McDowell, MD, ${ }^{1}$ William C. Newman, MD, ${ }^{1}$ Gary E. Mason, MD, MS, ${ }^{2}$ \\ Stephanie Greene, MD, ${ }^{1}$ and Mandeep S. Tamber, MD, PhD'1

\begin{abstract}
${ }^{1}$ Department of Neurological Surgery, and 2Division of Pediatric Hematology/Oncology, Children's Hospital of Pittsburgh, University of Pittsburgh, Pennsylvania
\end{abstract}

\begin{abstract}
Nivolumab is an immune checkpoint inhibitor $(\mathrm{ICl})$ currently undergoing Phase III clinical trials for the treatment of glioblastoma. The authors present the case of a 10-year-old girl with glioblastoma treated with nivolumab under compassionate-use guidelines. After the first dose of nivolumab the patient developed hemiparesis, cerebral edema, and significant midline shift due to severe tumor necrosis. She was managed using intravenous dexamethasone and discharged on a dexamethasone taper. The patient's condition rapidly deteriorated after the second dose of nivolumab, demonstrating hemiplegia, seizures, and eventually unresponsiveness with a fixed and dilated left pupil. Computed tomography of her brain revealed malignant cerebral edema requiring emergency decompressive hemicraniectomy. Repeat imaging demonstrated increased size of the lesion, reflecting immune-mediated inflammation and tumor necrosis. The patient remained densely hemiplegic, but became progressively more interactive and was ultimately extubated. She resumed nivolumab several weeks later, but again her condition deteriorated with headache, vomiting, swelling at the craniectomy site, and limited right-sided facial movement following the sixth dose. MRI demonstrated severe midline shift and uncal herniation despite her craniectomy. Her condition gradually declined, and she died several days later under "do not resuscitate/do not intubate" orders. To the authors' knowledge, this represents the first case of malignant cerebral edema requiring operative intervention following nivolumab treatment for glioblastoma in a pediatric patient.
\end{abstract}

https://thejns.org/doi/abs/10.3171/2016.8.PEDS16326

KEY WORDS glioblastoma; adverse event; hemicraniectomy; nivolumab; oncology

$\mathrm{P}$ EDIATRIC glioblastoma is a rare and fatal condition that is an area of intense investigation designed to identify potentially life-prolonging therapeutics. A Phase III clinical trial utilizing nivolumab in the treatment of pediatric glioblastoma is actively under way that potentially may induce powerful immune responses in the perilesional region. The authors present the case of a 10 -year-old girl with repeated episodes of malignant cerebral edema associated with administration of nivolumab that resulted in hemiplegia, seizures, and decline in mental status.

\section{Case Report}

History and Examination

The patient in this case was a 10-year-old girl with a history of a biallelic PMS2 mismatch repair deficiency - with
2 deceased siblings with the same mutation-who initially underwent resection of a left frontoparietal glioblastoma without systemic chemotherapy or radiation treatment. She underwent repeat resection for recurrence 7 months later with pathological results consistent with glioblastoma. One month later she presented to the emergency department (ED) with new-onset seizures and mood changes. MRI demonstrated tumor recurrence (Fig. 1). Her family declined standard chemotherapy, isotretinoin, and radiation, and instead pursued immunotherapy with nivolumab under compassionate-use guidelines. Extensive discussions of this experimental treatment were held, and given the family's two prior experiences with this syndrome resulting in morbidity and death despite standard of care treatment, only advances beyond the traditional regimens prescribed were of interest to them. Possible inflammatory

ABBREVIATIONS ED = emergency department; ICI = immune checkpoint inhibitor; NSCLC = non-small cell lung cancer; $\mathrm{PCA}=$ posterior cerebral artery; PD1 = programmed cell death protein $1 ; \mathrm{RCC}=$ renal cell carcinoma; $\mathrm{SRS}=$ stereotactic radiosurgery.

SUBMITTED June 5, 2016. ACCEPTED August 16, 2016.

INCLUDE WHEN CITING Published online November 18, 2016; DOI: 10.3171/2016.8.PEDS16326. 

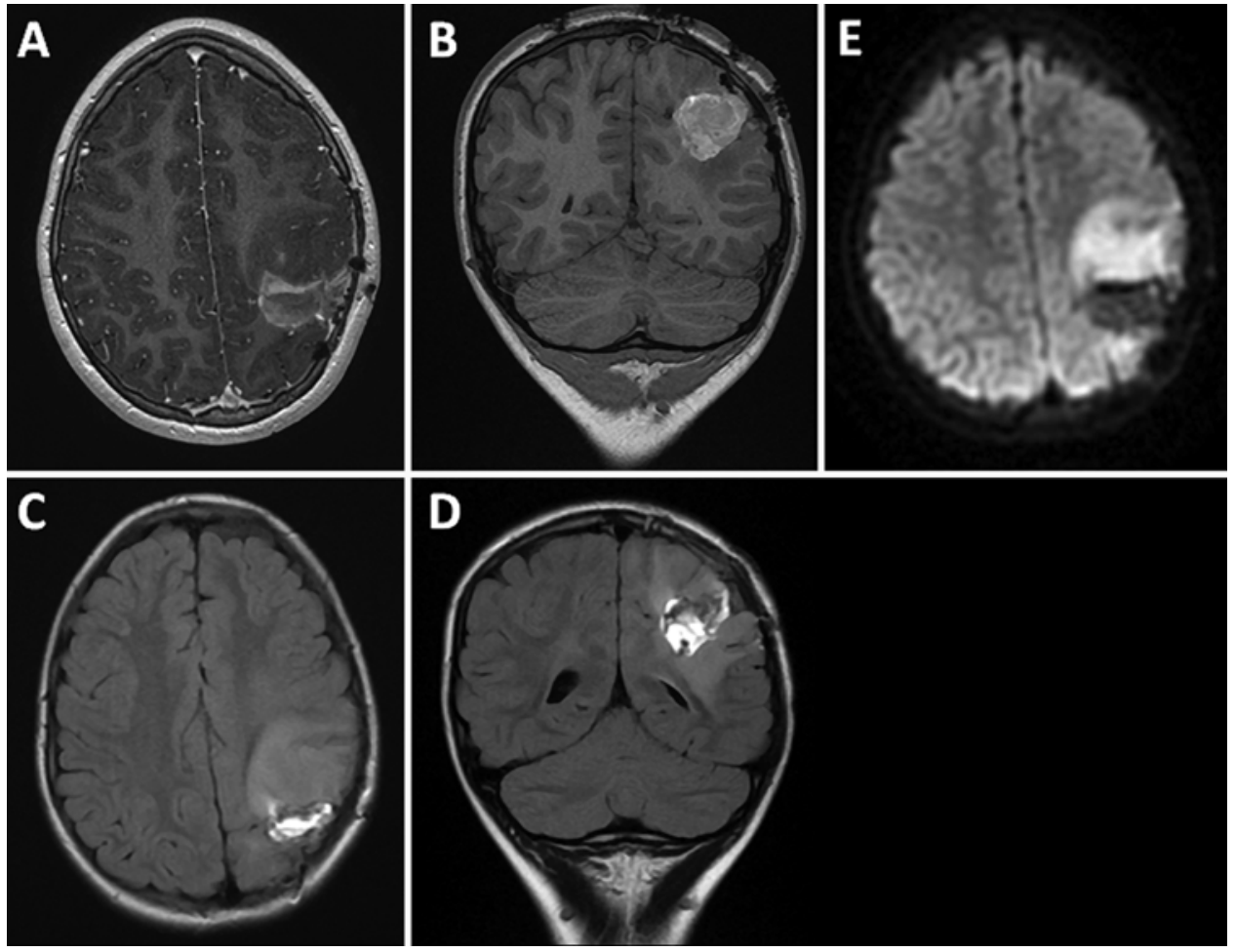

FIG. 1. MR images of the patient's brain demonstrating a left frontoparietal glioblastoma prior to nivolumab treatment: axial T1weighted with contrast administration (A), coronal T1-weighted with contrast administration (B), axial FLAIR (C), coronal FLAIR (D), and axial diffusion-weighted (E) images.

responses to the drug were discussed specifically with the family, who accepted the risks given the potential for aggressive tumor response.

\section{Initial Nivolumab Infusion}

Approximately 2 weeks after her initial nivolumab intravenous infusion, the patient presented to the ED with lethargy, irritability, emesis, worsening headache, and a right-sided hemiparesis. CT demonstrated a left-frontal, mixed-density lesion with surrounding edema and significant midline shift concerning for severe tumor necrosis (Fig. 2). She was admitted and treated with intravenous dexamethasone. Her hemiparesis and other symptoms improved, and she was discharged after 2 days on a dexamethasone taper.

\section{Second Nivolumab Infusion}

Two weeks later, she received her second infusion of nivolumab in the morning. She had a rapid onset of discomfort, lethargy, and vomiting that gradually worsened as the day progressed. Approximately 12 hours after infusion, she was unable to get out of the bathtub by herself, experienced a tonic-clonic seizure, and was shortly thereafter found to be unresponsive with a dilated left pupil. On arrival to the ED at about 1:00 AM, approximately 16 hours after infusion, she was intubated and given $1 \mathrm{mg} / \mathrm{kg}$ of mannitol and $10 \mathrm{mg}$ of dexamethasone, resulting in a return of her pupillary function. Her physical examination was significant for spontaneous movement of her left side with a right hemiplegia. CT of her brain demonstrated increased size of her mixed-density left frontoparietal lesion with increased midline shift and hydrocephalus (Fig. 2). Detailed goals of her care were discussed with the family. The family acknowledged a strong reluctance for any additional treatment that might result in neurological decline, given their past two experiences with fatal disease of the same etiology. However, the acuity of the situation prompted the patient's parents to request surgical decompression due to its lifesaving benefit, despite the risk of neurological deficits.

\section{Operation}

The patient was taken to the operating room for emergency decompressive hemicraniectomy and placement of
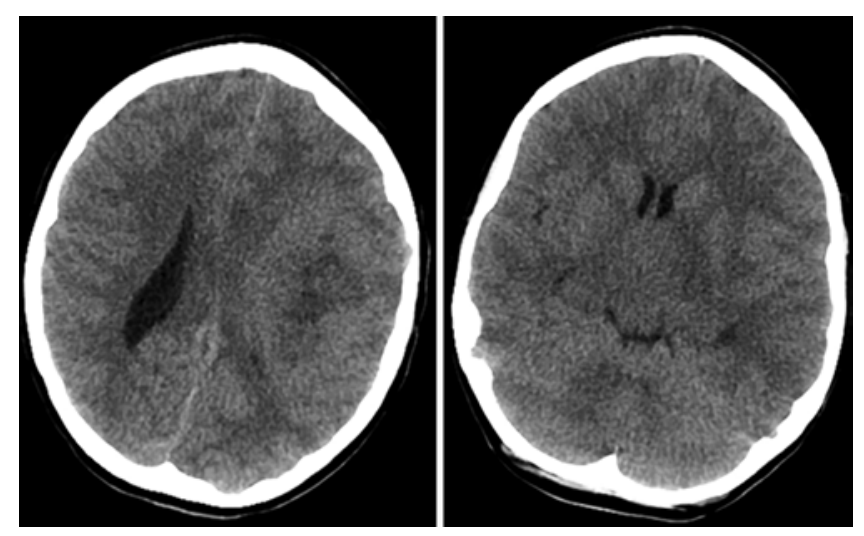

FIG. 2. Axial CT scans of the head demonstrating moderate cerebral edema following the first nivolumab dose. 

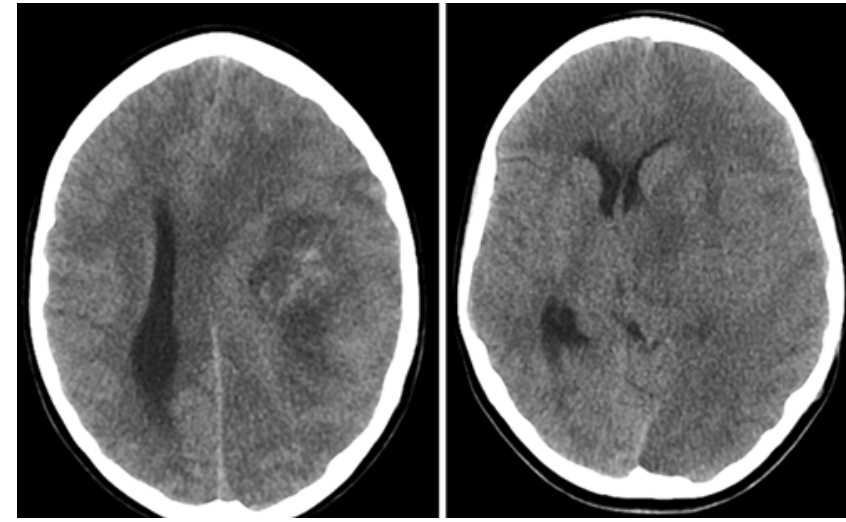

FIG. 3. Axial CT scans of the head demonstrating severe cerebral edema following a second nivolumab dose.

an external ventricular drain (Fig. 3). On immediate postoperative examination, the patient was following commands on her left side with persistent right hemiplegia. Repeat MRI demonstrated a predominantly hypointense lesion with significant mass effect, believed to represent treatment effect with tumor necrosis, as well as uncal herniation and a left posterior cerebral artery (PCA) infarct (Fig. 3). The patient was able to be extubated several days later without need for reintubation.

\section{Resumption of Nivolumab Treatment}

The patient demonstrated improved mental status and motor skills upon readmission several weeks later and resumed nivolumab treatment. The rationale to continue nivolumab despite her past two adverse reactions can be found in a recent article by Bouffet et al., which describes the course of nivolumab treatment for 2 pediatric patients with glioblastoma caused by biallelic PMS2 mismatch repair deficiency. ${ }^{3}$ While these cases also demonstrated transient cerebral edema due to nivolumab treatments, they were successfully managed with dexamethasone. Neither case required operative intervention, and, in contrast to this case, repeated doses were found to result in decreased cerebral edema. One patient ultimately demonstrated complete tumor resolution, and the other experienced significant tumor reduction. The authors cautioned against misinterpretation of the posttreatment flare as an adverse reaction to ICIs, and instead suggested that it represented a positive response and local inflammation secondary to Tcell activation. Rather than management using prolonged courses of immunosuppressive drugs or premature treatment cessation, the authors recommended a rapid corticosteroid taper along with continued treatment to allow a delayed clinical response. This information was discussed with the family, and, with acknowledgment of the risks, they chose to continue nivolumab treatments despite the adverse responses. Adjuvant treatment options were again offered to the family, but were declined for the same reasons discussed above. The patient was cleared by neurooncology to continue taking nivolumab, and was stable until a month later when she presented with headache, vomiting, craniectomy site swelling, and limited right facial movement just hours after receiving the sixth dose. MRI dem-
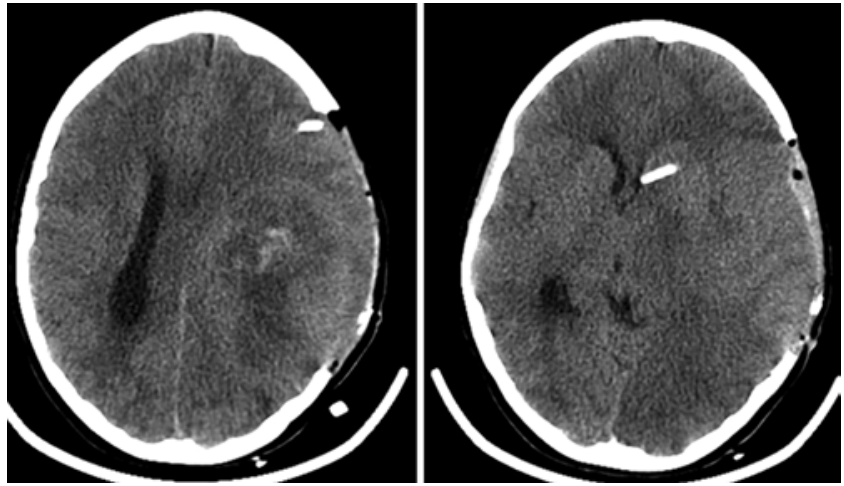

FIG. 4. Axial CT scans after a decompressive hemicraniectomy.

onstrated severe midline shift and uncal herniation despite her craniectomy (Fig. 4). She was provided supportive care; however, she gradually worsened and suffered a seizure, along with clinical signs of active uncal and subfalcine herniation. She was allowed to die the following day under "do not resuscitate/do not intubate" instructions.

\section{Autopsy Results}

The autopsy revealed a large, left, parietal glioblastoma as the cause of death. The tumor extensively involved the left cerebrum and corpus callosum, and protruded 11.5 $\times 9 \times 2 \mathrm{~cm}$ from the craniectomy site. It also extended down the corticospinal tract to involve both the midbrain and pons, along with associated axonal degeneration of the medullary pyramids and corticospinal tract. There was some necrosis but no atypical inflammation noted on histopathology, yet a high rate of mitotic activity and a markedly high Ki 67 proliferation index were observed diffusely (Fig. 5). Further examination revealed left uncal and subfalcine herniation, as well as subacute infarctions in both the right anterior cerebral artery and left PCA territories. The patient's death was reported by the presiding neurooncologist as a serious adverse event to the pharmaceutical sponsor and the appropriate governing authority overseeing the Children's Hospital of Pittsburgh.

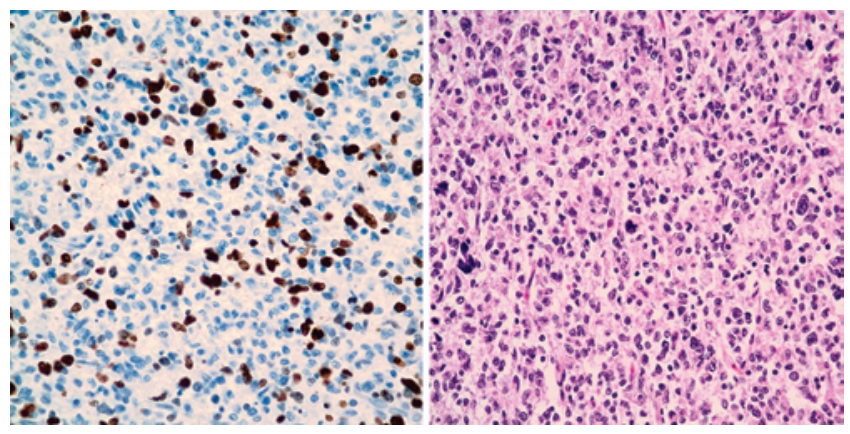

FIG. 5. Photomicrographs of histopathology slides of brain sections from autopsy. Left: Mitotically active, densely cellular tumor was noted at autopsy, but there was little necrosis or endothelial proliferation. Original magnification $\times 400$. Right: The Ki 67 proliferation index was markedly high. There were no perivascular inflammatory infiltrates. Original magnification $\times 400$. Figure is available in color online only. 


\section{Discussion}

The diagnosis of glioblastoma in pediatric patients is rare, accounting for only $3 \%$ of childhood CNS tumors. ${ }^{15}$ Secondary glioblastoma has frequently been documented to progress from preexisting astrocytomas in pediatric patients, but represents only $5.3 \%-8.8 \%$ of all glioblastomas, and carries a median overall survival of 24-31 months following standard treatment. ${ }^{12}$ Despite recent advances in treatment options, glioblastoma remains a disease that requires much study. The current standard of care for adults with glioblastoma involves aggressive resection, followed by temozolomide and radiotherapy; however, multiple studies have shown that temozolomide failed to improve outcomes in children. ${ }^{4,6,10,13}$ Researchers hypothesize that this failure may be due to tumor overexpression of the DNA repair enzyme $\mathrm{O}^{6}$-methylguanineDNA methyltransferase. ${ }^{4,6,10}$ Immunotherapy with ICIs, such as those targeting programmed cell death protein 1 (PD1), like nivolumab and pembrolizumab, or cytotoxic T-lymphocyte-associated antigen 4, like ipilimumab, hold promise as new therapeutic approaches for managing glioblastoma in pediatric patients.

Nivolumab is currently FDA-approved for metastatic melanoma, non-small cell lung cancer (NSCLC), renal cell carcinoma (RCC), and is currently undergoing Phase III clinical trials (the CheckMate-143 trial) for glioblastoma. ${ }^{11,13}$ In clinical trials documenting the safety profile of nivolumab in treating melanoma, NSCLC, and RCC, malignant cerebral edema was not among its adverse events. ${ }^{13,16}$ Moreover, preliminary data from a small cohort of patients from CheckMate-143 documenting the safety and tolerability of nivolumab in treating glioblastoma showed that of 10 patients treated with nivolumab monotherapy at $3 \mathrm{mg} / \mathrm{kg}$ every other week, all adverse events were Grade $1-2$, with fatigue $(n=3)$ and nausea $(n=3)$ the most common adverse events. ${ }^{14}$ While these and other studies appear to demonstrate the safety of nivolumab in treating a wide variety of malignancies, our patient demonstrated severe cerebral edema following treatment with nivolumab. ${ }^{3}$

To our knowledge, this is the first report of malignant cerebral edema and severely elevated intracranial pressures requiring emergency decompression in a pediatric patient with glioblastoma treated with a PD1 inhibitor. Her underlying genetic defect likely contributed to the severity of her reaction to nivolumab. Biallelic mismatch repair mutation cancers demonstrate an extremely high degree of mutation and microsatellite instability. ${ }^{3,5}$ This hypermutation increases the likelihood that the cancer possesses or will create neoantigens that activate T-cells. ${ }^{3}$ In our patient, augmented baseline T-cell responsiveness to her glioblastoma compounded by nivolumab-mediated T-cell activation could explain her malignant cerebral edema and herniation events. However, 2 other pediatric patients who also harbored biallelic PMS2 mismatch repair-deficient glioblastoma did not experience nearly the same degree of inflammatory response to nivolumab as our patient. ${ }^{3}$ In fact, successive nivolumab treatments reduced cerebral edema and ultimately resulted in tumor shrinkage, suggesting that the response pattern or severity may not be consistent among patients with the same genetic defect.
Chemotherapy-induced cerebral edema is a poorly documented phenomenon. This is especially true for ICIs, which represent a new treatment modality. In a retrospective analysis of 26 patients with melanoma brain metastases treated with both stereotactic radiosurgery (SRS) and nivolumab, Ahmed et al. found 6 patients $(23 \%)$ with $\geq$ $20 \%$ tumor volume increase due to cerebral edema. ${ }^{1}$ In a similar study by Kiess et al. documenting clinical outcomes of 46 patients with melanoma brain metastases treated with a combination of SRS and ipilimumab, a staggering $82 \%$ had follow-up imaging consistent with hemorrhage, edema, or both. ${ }^{8}$ While it is difficult to discern individual contributions of SRS and ICIs to cerebral edema, most cases of cerebral edema occurred after initiating ICI therapy. ${ }^{1,8}$ Moreover, a randomized controlled trial involving 67 patients with brain metastases treated with SRS alone resulted in only 3 patients (4.5\%) with any late treatment-related neurological toxicity. ${ }^{2}$ The low reported frequency of treatment-related neurotoxicity with SRS alone is in stark contrast to data reported by Ahmed et al. and Kiess et al., which suggest that ICIs may precipitate cerebral edema. Nevertheless, cerebral edema developed subacutely in the cases treated with SRS alone, unlike the acute presentation in our patient, and none were severe enough to merit surgical intervention. Some tumor necrosis was noted on autopsy, but the lack of severe atypical inflammation suggests that tumor progression may also have played a role.

Several reports of cerebral edema following ipilimumab monotherapy have been documented. In a retrospective analysis of a Phase II trial for ipilimumab in treating melanoma patients with brain metastases, Weber et al. found that of 16 patients, 1 had cerebral edema that was successfully managed with dexamethasone. ${ }^{17}$ Another case demonstrated immune-mediated inflammation and cerebral edema secondary to extensive tumor necrosis in a woman treated with ipilimumab for advanced melanoma metastases to the brain. ${ }^{7}$ Like the patient in the retrospective study, the edema and associated neurological deficits in this woman were also resolved with dexamethasone.

An autopsy study by Koelzer et al. of a patient with metastatic melanoma treated with ipilimumab followed by nivolumab revealed systemic inflammation involving the lungs, heart, CNS, liver, and bone marrow, implying the presence of undetected widespread inflammation. ${ }^{9}$ However, this was not the case in our patient on autopsy, suggesting that the immunomodulatory effects were localized to the tumor with minimal parenchymal inflammation. In a recent review article, Preusser et al. noted that one of the main challenges facing immunotherapy for managing CNS tumors is balancing treatment efficacy with cerebral edema and elevated intracranial pressures due to an augmented inflammatory response against the tumor. ${ }^{13}$ While most cases of ICI-induced cerebral edema were either mild or asymptomatic, and could be successfully managed with corticosteroids, our patient's condition deteriorated rapidly following each nivolumab dose and presented with malignant cerebral edema along with sequelae of herniation and infarction. These cases demonstrate a potential risk of utilizing ICIs in the treatment of pediatric CNS neoplasms, and highlight the need for further clinical investigation. 


\section{Conclusions}

Nivolumab is a powerful chemotherapeutic agent in pediatric patients with secondary glioblastoma. However, strong treatment response may result in rapid inflammation and tissue breakdown, leading to malignant cerebral edema and herniation events. Given the potential severity of side effects, further consideration of the risk of malignant cerebral edema in these patients is needed.

\section{Acknowledgments}

We would like to thank Dr. Ronald Hamilton and Dr. Jason Chiang for providing histopathological samples for this report.

\section{References}

1. Ahmed KA, Stallworth DG, Kim Y, Johnstone PA, Harrison LB, Caudell JJ, et al: Clinical outcomes of melanoma brain metastases treated with stereotactic radiation and anti-PD-1 therapy. Ann Oncol 27:434-441, 2016

2. Aoyama H, Shirato H, Tago M, Nakagawa K, Toyoda T, Hatano K, et al: Stereotactic radiosurgery plus whole-brain radiation therapy vs stereotactic radiosurgery alone for treatment of brain metastases: a randomized controlled trial. JAMA 295:2483-2491, 2006

3. Bouffet E, Larouche V, Campbell BB, Merico D, de Borja $\mathrm{R}$, Aronson M, et al: Immune checkpoint inhibition for hypermutant glioblastoma multiforme resulting from germline biallelic mismatch repair deficiency. J Clin Oncol 34:22062211, 2016

4. Cohen KJ, Pollack IF, Zhou T, Buxton A, Holmes EJ, Burger PC, et al: Temozolomide in the treatment of high-grade gliomas in children: a report from the Children's Oncology Group. Neuro Oncol 13:317-323, 2011

5. Dudley JC, Lin MT, Le DT, Eshleman JR: Microsatellite instability as a biomarker for PD-1 blockade. Clin Cancer Res 22:813-820, 2016

6. Fangusaro J: Pediatric high grade glioma: a review and update on tumor clinical characteristics and biology. Front Oncol 2:105, 2012

7. Hodi FS, Oble DA, Drappatz J, Velazquez EF, Ramaiya N, Ramakrishna N, et al: CTLA-4 blockade with ipilimumab induces significant clinical benefit in a female with melanoma metastases to the CNS. Nat Clin Pract Oncol 5:557-561, 2008

8. Kiess AP, Wolchok JD, Barker CA, Postow MA, Tabar V, Huse JT, et al: Stereotactic radiosurgery for melanoma brain metastases in patients receiving ipilimumab: safety profile and efficacy of combined treatment. Int J Radiat Oncol Biol Phys 92:368-375, 2015

9. Koelzer VH, Rothschild SI, Zihler D, Wicki A, Willi B, Willi N, et al: Systemic inflammation in a melanoma patient treated with immune checkpoint inhibitors-an autopsy study. J Immunother Cancer 4:13, 2016

10. MacDonald TJ, Aguilera D, Kramm CM: Treatment of highgrade glioma in children and adolescents. Neuro Oncol 13:1049-1058, 2011

11. Motzer RJ, Rini BI, McDermott DF, Redman BG, Kuzel TM, Harrison MR, et al: Nivolumab for metastatic renal cell carcinoma: results of a randomized phase II trial. J Clin Oncol 33:1430-1437, 2015

12. Ohgaki H, Kleihues P: The definition of primary and secondary glioblastoma. Clin Cancer Res 19:764-772, 2013

13. Preusser M, Lim M, Hafler DA, Reardon DA, Sampson JH: Prospects of immune checkpoint modulators in the treatment of glioblastoma. Nat Rev Neurol 11:504-514, 2015

14. Sampson JH, Vlahovic G, Sahebjam S, Omuro AMP, Baehring JM, Hafler DA, et al: Preliminary safety and activity of nivolumab and its combination with ipilimumab in recurrent glioblastoma (GBM): CHECKMATE-143. Presented at the 2015 ASCO Annual Meeting, Chicago, 2015 (Abstract) (http://meetinglibrary.asco.org/content/147328-156) [Accessed September 16, 2016]

15. Song KS, Phi JH, Cho BK, Wang KC, Lee JY, Kim DG, et al: Long-term outcomes in children with glioblastoma. J Neurosurg Pediatr 6:145-149, 2010

16. Topalian SL, Hodi FS, Brahmer JR, Gettinger SN, Smith DC, McDermott DF, et al: Safety, activity, and immune correlates of anti-PD-1 antibody in cancer. N Engl J Med 366:2443-2454, 2012

17. Weber JS, Amin A, Minor D, Siegel J, Berman D, O’Day SJ: Safety and clinical activity of ipilimumab in melanoma patients with brain metastases: retrospective analysis of data from a phase 2 trial. Melanoma Res 21:530-534, 2011

\section{Disclosures}

The authors report no conflict of interest concerning the materials or methods used in this study or the findings specified in this paper.

\section{Author Contributions}

Conception and design: McDowell. Acquisition of data: McDowell, Zhu. Analysis and interpretation of data: all authors. Drafting the article: all authors. Critically revising the article: all authors. Reviewed submitted version of manuscript: all authors. Approved the final version of the manuscript on behalf of all authors: McDowell. Administrative/technical/material support: Tamber. Study supervision: Tamber.

\section{Correspondence}

Michael M. McDowell, University of Pittsburgh, Neurological Surgery, 200 Lothrop St., Pittsburgh, PA 15213. email: mcdowellmm2@upmc.edu. 